\title{
Pathogenic Variation of Phakopsora pachyrhizi Infecting Soybean in Nigeria
}

\author{
M. Twizeyimana, P. S. Ojiambo, K. Sonder, T. Ikotun, G. L. Hartman, and R. Bandyopadhyay
}

First author: Department of Crop Protection and Environmental Biology, University of Ibadan, Ibadan, Nigeria and International Institute of Tropical Agriculture (IITA), PMB 5320, Ibadan, Nigeria; second author: Department of Plant Pathology, North Carolina State University, Raleigh, NC 27695; third author: Geo-Spatial Laboratory, IITA, PMB 5320, Ibadan, Nigeria; fourth author: Department of Crop Protection and Environmental Biology, University of Ibadan, Ibadan, Nigeria; fifth author: U.S. Department of Agriculture-Agricultural Research Service and Department of Crop Sciences, University of Illinois, Urbana, IL 61801; and sixth author: IITA, PMB 5320, Ibadan, Nigeria.

Accepted for publication 14 November 2008.

\begin{abstract}
Twizeyimana, M., Ojiambo, P. S., Sonder, K., Ikotun, T., Hartman, G. L., and Bandyopadhyay, R. 2009. Pathogenic variation of Phakopsora pachyrhizi infecting soybean in Nigeria. Phytopathology 99:353-361.

Soybean rust, caused by Phakopsora pachyrhizi, is an important disease in Nigeria and many other soybean-producing countries worldwide. To determine the geographical distribution of soybean rust in Nigeria, soybean fields were surveyed in the Derived Savanna (DS), Northern Guinea Savanna (NGS), and Southern Guinea Savanna (SGS) agroecological zones in Nigeria between 2004 and 2006. Disease severity in each zone was determined and analyzed using geostatistics. Prevalence of infected fields and disease severity in surveyed fields were significantly $(P<0.05)$ different between geographical zones with both variables being higher in the DS zone than in either NGS or SGS zones. Geostatistical analysis indicated that the spatial influence of disease severity at one location on severity at other locations was between 75 and $120 \mathrm{~km}$. An exponential model best described the relationship between semivariance and lag distance when rust severity was high. Spatial

detached soybean leaves. To establish the nature of pathogenic variation in $P$. pachyrhizi, a set of four soybean accessions with $R p p_{1}, R p p_{2}, R p p_{3}$, and $R p p_{4}$ resistance genes, two highly resistant and two highly susceptible genotypes were inoculated with single uredinial isolates. Principal component analysis on the number of uredinia per square centimeter of leaf tissue for 116 isolates indicated that an adequate summary of pathogenic variation was obtained using only four genotypes. Of these four, PI 459025B (with $R p p_{4}$ gene) and TG× 1485-1D had the lowest and highest number of uredinia per square centimeter, respectively. Based on cluster analysis of the number of uredinia per square centimeter, seven pathotype clusters were determined. Isolates in cluster III were the most virulent, while those in cluster IV were the least virulent. Shannon's index $(H)$ revealed a more diverse pathogen population in the DS zone $(H=1.21)$ compared to the rust population in SGS and NGS with $H$ values of 1.08 and 0.91 , respectively. This work will be useful in breeding and management of soybean rust by facilitating identification of resistant genotypes and targeting cultivars with specific resistance to match prevailing $P$. pachyrhizi pathotypes in a given geographical zone.
\end{abstract} interpolation of rust severity showed that locations in the DS zone were more conducive for the rust epidemic compared to areas in the NGS zone. In the 2005 survey, 116 purified isolates were established in culture on

Soybean rust, caused by Phakopsora pachyrhizi (H. Sydow \& Sydow), is one of the most economically important foliar diseases affecting soybean (Glycine max [L.] Merr.) worldwide (18) causing yield losses from 13 to $80 \%$ (31). In absence of control measures, complete crop loss is not uncommon (31). Soybean, which is native to China where it has been cultivated since the 11th century (19), is a recent introduction to Africa. Presently, South America is the largest producer of soybean accounting for nearly $45 \%$ of the total world production followed by North America and Asia with 41 and $12 \%$, respectively, of the total production (14). Although Africa accounts for only $1 \%$ of the world soybean production, there is potential to increase production and currently Nigeria accounts for the largest production share on the continent (14). Most of the soybean production in Nigeria is concentrated in the savannas where the crop has contributed to substantial social, economic and environmental

Corresponding author: R. Bandyopadhyay

E-mail address: r.bandyopadhyay@ cgiar.org

doi:10.1094/PHYTO-99-4-0353

This article is in the public domain and not copyrightable. It may be freely reprinted with customary crediting of the source. The American Phytopathological Society, 2009.
Additional keywords: pathogen diversity, pathotype composition, virulence analysis, West Africa.

benefits (27). Soybean rust was first recorded in Oyo state of Nigeria in 1999 (2), and is now endemic in major soybean-growing areas in the country. However, the relative distribution and importance of the disease in different regions of the Nigerian savannas remains undocumented. Geostatistical tools offer opportunities to describe spatial patterns of diseases and relate these patterns to various factors that affect disease development (36).

A pathotype is a class of pathogen genotypes whose members express the same pattern of host-specific virulence towards individual clones or species within a specified set (26). Virulence is the relative ability of a pathogen with a given genotype to cause damage on a specific host genotype or the ability of a pathotype to multiply on a host cultivar containing genes conferring resistance to other pathotypes of that pathogen (28). Therefore, a pathotype can be a heterogeneous group of genotypes having in common only the same pattern of species-specific virulence for a given set of species. The pathotype structure for a pathogen population reflects the present structuring of the population with respect to classification of species-specific virulence, which includes the influence of the present structuring of the pathotypespecific resistance for the plant population (26). Thus, pathotyping is a means of monitoring the present state of interaction between pathogen virulence and plant resistance for a pathogen population and host-plant population. 
The genetic plasticity of $P$. pachyrhizi populations has often resulted in the development of virulent strains following the release of rust-resistant cultivars, particularly those with $R p p_{1}$ and $R p p_{3}$ resistance genes (18). The cultivar Komata (with $R p p_{1}$ ) was identified as resistant in germplasm evaluations done during 1961 to 1963 (5). By 1966, susceptible lesions were found on plants of Komata in field trials, and by the mid-1970s the line was not considered as a useful source of resistance (21). Similarly, the accession PI 230970 (with $R p p_{2}$ ) was identified as resistant in field evaluations from 1971 to 1973 , but by 1976 a few tan colored lesions were observed on plants in the field (5). The resistance in Ankur (with $R p p_{3}$ ), identified in the early 1970s was ineffective in the late 1970s (38), providing another example of the ability of $P$. pachyrhizi to overcome single-gene resistance. A recent study (4) indicates that selections from Bing Nan (with $R p p_{4}$ ) are now susceptible to $P$. pachyrhizi.

Studies on pathogen variation on $P$. pachyrhizi isolates on soybean have exclusively focused on qualitative differences in virulence $(4,6,31,37)$. This qualitative nature of virulence is based on three infection types on soybean (6): (i) RB, red-brown lesions with either no uredinia or only sparsely sporulating uredinia; (ii) TAN, tan lesions with many uredinia and abundant sporulation, and (iii) HR, immune, no visible infection. Bonde et al. (4) reported that intermediate reactions were difficult to score based on the current RB/TAN designation, and a quantitative approach to assess pathogen virulence was needed. In addition, pathogen diversity demonstrated by quantitative virulence, i.e., quantitative differences in pathogen development on a given host such as number and sizes of uredinia (6), can also be useful in breeding for general (minor gene) resistance in soybean. Recently, Bonde et al. (4) suggested that this quantitative difference in virulence could detect both major and partial resistance to soybean rust. Partial resistance is a form of incomplete resistance characterized by a reduced rate of epidemic development. In this study, we adopted the number of uredinia per unit leaf area as a quantitative measurement of virulence of the pathogen (25).

In West Africa, considerable variation in rust resistance among soybean genotypes at different sites has been reported (34), pointing to a variable composition in the pathogen population at these sites. Further, an entire differential set from the Asian Vegetable Research and Development Centre (AVRDC) recommended for identification of rust races (33) was reported to be susceptible to isolates from South Africa (8), providing evidence of a diverse rust population in Africa. However, analysis of pathogen diversity is still lacking for $P$. pachyrhizi infecting soybean in the region. We are also unaware of any study in the literature where pathogenic variation of $P$. pachyrhizi has been based on quantitative differences in virulence. In addition, there is no inventory of dominant pathotypes and their geographical distribution to better design germplasm evaluation and cultivar development breeding programs in West Africa. Based on these considerations, this study was conducted to (i) analyze the spatial distribution of the soybean rust in Nigeria; (ii) determine a host differential set for identifying dominant pathotypes of $P$. pachyrhizi infecting soybean in Nigeria; and (iii) examine the geographical distribution of $P$. pachyrhizi pathotypes in main soybean production zones in Nigeria.

\section{MATERIALS AND METHODS}

Field surveys and analysis of survey data. Fields were surveyed for soybean rust from 2004 to 2006 in soybean production areas in three agroecological zones in Nigeria; Derived Savanna (DS), Southern Guinea Savanna (SGS), and Northern Guinea Savanna (NGS) (22). A total of 11, 6, and 3 states were covered during the survey in DS, SGS, and NGS zones, respectively. Surveys were conducted between late October and early November of each year when plants had seeds in pods that were fully developed (R6 stage). Specifically, 72, 29, and 25 fields were surveyed in DS, SGS, and NGS, respectively, in 2004. In 2005, 58,26 , and 41 fields were surveyed in DS, SGS, and NGS, respectively, while in 2006, 65, 38, and 38 fields were surveyed in DS, SGS, and NGS, respectively. The fields were a mixture of smallholder and commercial plots with an average size of 0.5 ha and characterized by very limited fungicide use. The DS lies within latitudes $6^{\circ} 8^{\prime}$ and $9^{\circ} 30^{\prime} \mathrm{N}$ and longitudes $2^{\circ} 40^{\prime}$ and $12^{\circ} 15^{\prime}$ $\mathrm{E}$ and has a bimodal rainfall distribution averaging from $1,300 \mathrm{~mm}$ to $1,500 \mathrm{~mm}$ annually, and maximum temperatures varying from 25 to $35^{\circ} \mathrm{C}$. The SGS zone lies within latitudes $8^{\circ} 4^{\prime}$ and $11^{\circ} 3^{\prime} \mathrm{N}$ and longitudes $2^{\circ} 41^{\prime}$ and $13^{\circ} 33^{\prime} \mathrm{E}$, with a bimodal rainfall averaging from $1,000 \mathrm{~mm}$ to $1,300 \mathrm{~mm}$ per year, and maximum temperatures ranging from 26 to $38^{\circ} \mathrm{C}$. The NGS lies within latitudes $9^{\circ} 10^{\prime}$ and $11^{\circ} 59^{\prime} \mathrm{N}$ and longitudes $3^{\circ} 19^{\prime}$ and $13^{\circ} 37^{\prime} \mathrm{E}$ and has a unimodal rainfall distribution averaging from $900 \mathrm{~mm}$ to $1,000 \mathrm{~mm}$ annually, and maximum temperatures varying from 28 to $40^{\circ} \mathrm{C}$. Across the regions, temperatures increase and rainfall decreases with increasing latitude with the DS furthest south followed by the SGS and the NGS in the north. Due to crop rotation practices, the same farm was not surveyed every year during the 3-year period but as much as possible farms surveyed in a locality were within proximity. For each field sampled, longitude, latitude, and elevation data were recorded using a hand-held Global Positioning System device (eTrex GPS, Garmin Corporation, Olathe, KS). To determine disease severity in each field, 20 plants were sampled for disease in a W-shaped sampling pattern. To facilitate disease assessment along the sampling transect, whole plants were divided into lower, intermediate, and upper sections. Each section was assessed for leaf area infected using a 0 to 9 scale (24) and the mean score of the three sections represented the disease severity score for the whole plant.

Out of the total number of fields surveyed for the presence of soybean rust, 62, 20, and 16 fields were infected in DS, SGS, and NGS zones, respectively, in 2004. In 2005, 52, 16, and 39 fields were infected in DS, SGS, and NGS zones, respectively, while in 2006, 51, 15, and 25 fields were infected in DS, SGS, and NGS zones, respectively. Based on the total number of fields surveyed and the corresponding number of infected fields, disease prevalence was calculated for each agroecological zone and separately for each year as the percentage of infected fields relative to the total number of fields surveyed. Disease severity data collected during the survey using the 0 to 9 scale were converted to percentages using the mid-point method (9). In each year, disease severity in each agroecological zone was calculated as the mean disease severity of all fields surveyed within their respective zones. Data on disease prevalence and disease severity were then subjected to analysis of variance (ANOVA) in which years and agroecological zones were considered as blocking and treatment variables, respectively. Differences in disease prevalence and disease severity between agroecological zones were determined using Fisher's protected least significant difference (LSD) test. All analyses were performed in SAS (version 9.1; SAS Institute, Cary, NC).

According to the latitude and longitude measured at each field, a point-coverage was generated for all fields in a geographic information system (GIS). Geo-referenced disease severity values were analyzed in the geostatistical analyst extension manager of ArcGIS version 9.2 (13). Semivariogram analysis was performed to describe the variance of disease severity and the spatial relationships between fields. Spatial structures were objectively compared by fitting exponential models to empirical variograms. The exponential model is a theoretical variogram model in which the semivariance rises smoothly from the first lag to a maximum stable value, i.e., sill, at a fixed range. The best fit exponential model was then used to estimate the nugget and the range of spatial dependency. Low nugget values can be interpreted as high level of aggregation of disease severity, while the range of spatial 
dependency is the maximum distance at which samples show spatial dependence. Using this kriging function, spatial distribution grids of rust severity were generated using severity data from all the surveyed fields. Spatial grids of interpolated disease severity were delimited to within a $10-\mathrm{km}$ radius around rust infected fields and this resulted in three separate grids in each year of the survey.

Isolate collection and inoculation. A total of 116 isolates of $P$. pachyrhizi were collected for analysis of pathogenic variability during the survey in 2005. Uredinial collections of P. pachyrhizi were made from soybean leaves collected when surveying different locations in the three agroecological zones: DS (54 collections), NGS (35 collections), and SGS (27 collections). A handheld GPS was used during the survey to record the coordinates of isolate collection sites. A collection consisted of one to several leaves bearing uredinia from a single plant or cultivar. Approximately $20 \mathrm{mg}$ of urediniospores were collected from the leaves using a cyclone spore collector (G-R Manufacturing Company, Manhattan, KS) and placed in 1.8-ml cryogenic vials. The vials were stored in liquid nitrogen and transported to the IITA pathology laboratory in Ibadan. To purify the collection, urediniospores from each location were used to inoculate healthy detached leaves (35) of a highly susceptible soybean cultivar TG× 1485-1D grown in the greenhouse. Prior to inoculation, frozen spores were heat shocked at $40^{\circ} \mathrm{C}$ for $5 \mathrm{~min}$, and hydrated by incubating over water in an enclosed petri plate overnight (15). When uredinia started to erupt ( 9 to 13 days after inoculation), urediniospores from a single isolated uredinium (i.e., without any uredinia close by) were picked using a sharp sterile needle under a stereoscopic binocular at $40 \times$, and were mixed with $0.3 \mathrm{ml}$ of sterile water. Using a fine sterile brush, the resulting spore suspension was then spread onto new detached leaves in a petri dish. Single urediniospore isolates derived after three cycles of single uredinial transfers to generate pure cultures were multiplied on detached leaves (35) of TG $\times 1485-1 \mathrm{D}$ to produce adequate spore population of each isolate for use in the subsequent pathogenic variation experiments.

Selection of host differentials. Eight soybean genotypes from USDA-ARS, National Agriculture Research Organization (NARO) in Uganda and IITA breeding program were selected to develop a differential set to classify $P$. pachyrhizi isolates. Criteria used for their selection were, prior knowledge of soybean rust resistance and differential reaction against $P$. pachyrhizi isolates. The accessions PI 200492 (with $R p p_{1}$ gene), PI $230970\left(R p p_{2}\right)$, PI 462312 $\left(R p p_{3}\right)$, PI $459025 \mathrm{~B}\left(R p p_{4}\right)$, and PI 594538A were from USDAARS. The accession PI 594538A has been reported to produce a TAN reaction when inoculated with a mixture of $P$. pachyrhizi isolates from Thailand, Brazil, Paraguay, and Zimbabwe (23) but had expressed only an HR reaction when inoculated with rust isolates from Nigeria (35). The variety UG-5 from NARO, which is resistant to rust in Uganda (20), expresses an RB reaction when inoculated with field isolates from Nigeria and Uganda. The soybean breeding lines TG $\times 1485-1 \mathrm{D}$ and TG $\times 1844-4 \mathrm{~F}$ from IITA breeding program were included due to their high susceptibility to rust (35) and they express a TAN reaction.

The putative host differential lines were evaluated for their reaction to the isolates using the detached leaf technique as described by Twizeyimana et al. (35). Plants of each of the eight differential lines were maintained in the greenhouse under rustfree conditions for a constant supply of 3 to 4 week old leaves for inoculation. Briefly, the abaxial leaf surfaces were sprayed with $400 \mu \mathrm{l}$ of spore suspension $\left(1 \times 10^{5}\right.$ spores $\left./ \mathrm{ml}\right)$ using an atomizer attached to an air compressor and a single leaf piece was carefully placed in a 9-cm-diameter petri dish with adaxial side appressed on $1 \%$ agar technical amended with kinetin at $10 \mu \mathrm{g} / \mathrm{ml}$. Petri dishes containing leaf pieces (two leaf pieces per dish and two replicate dishes for each isolate-differential combination) were then incubated at $20^{\circ} \mathrm{C}$ with $12 / 12 \mathrm{~h}$ light/dark cycle for 17 days after which lesion type was recorded and the number of sporu- lating uredinia per square centimeter of leaf tissue (henceforth referred to as uredinia per square centimeter) were counted as described by Twizeyimana et al. (35). Inoculations to obtain records of reaction types and uredinia per square centimeter were repeated once and each isolate treatment was replicated three times in a single run. Prior to analysis, number of uredinia per square centimeter from all isolates recorded from the two runs were subjected to ANOVA to test for homogeneity of error variance (16). No heterogeneity in the data was detected and data were pooled over runs for final analysis and presentation. Uredinia per square centimeter were averaged over replications to form observations representing a mean value for each isolate and first subjected to preliminary analysis using PROC UNIVARIATE in SAS to examine the distribution of uredinia per square centimeter. From this preliminary analysis, three uredinia classes were defined based on the departure from the grand mean for number of uredinia across all isolates and genotypes: the first, second, and third classes consisted of uredinia numbers that were one standard deviation above the grand mean, one standard deviation around the grand mean, and one standard deviation below the grand mean, respectively. The first class that defined a highly virulent category was assigned a value of 1 , while the second and third classes were assigned a value of 2 and 3, respectively. The latter two groups defined virulent and less virulent categories, respectively.

The multivariate structure of $P$. pachyrhizi isolates was examined by principal component analysis as described by Chakraborty et al. (11) using SAS. Uredinia classes (i.e., 1, 2, or 3 ) on each host differential were regarded as variables and each isolate was regarded as an observation. Principal component analysis was used to generate composite scores, which are linear combinations of the ratings on each accession. Principal component scores have the maximum variance of any set of uncorrelated normalized linear combinations of the original ratings. Each accession was dropped in turn, and the principal components were recomputed. The first four principal components scores were then rotated and translated into maximum conformity with the principal components based on the full set of differential hosts. For each differential host, the sum of squared distance between the new configuration (based on the reduced differential set) and the original four-component configuration was calculated and expressed as a percentage of the sum of squares of the original configuration. This "stress statistic" was calculated as each genotype was dropped in turn. The genotype with the minimum stress was then dropped permanently, and each of the remaining genotypes was dropped in turn. The process was repeated in a stepwise fashion, eliminating one differential host (corresponding to minimum stress) at each step. The minimum stress was plotted against the number of genotypes excluded in each step. The remaining genotypes were then included in a putative differential set.

Pathogenic variation and data analysis for pathotype assignment. The multivariate response across differentials was explored using SAS to determine the number of independent dimensions needed to account for variation in the response profile. Uredinia classes (i.e., 1, 2, or 3) as defined above for each differential, were considered separate variates. Principal component scores were calculated from the resulting data matrix of 116 observations (isolates) and eight variates (differentials). To determine the number of pathotype clusters, the cluster tree was examined with the number of clusters varying from 2 to 11 , and the proportional reduction in residual sum of squares $\left(R^{2}\right)$ was plotted against the number of clusters. Pathotype clusters were also determined using the average linkage method and clustering criteria and first two principal components were plotted to visualize the clustering of isolates.

Geographical distribution of pathotype clusters. The Shannon's index (29) was used to estimate pathogen diversity in 
the three agroecological zones for isolates collected in 2005. The index was calculated using the formula: $H(A)=-\sum\left[\left(n_{i} / N\right) \times\right.$ $\left.\ln \left(n_{i} / N\right)\right]$, where $n_{i}$ is the number of $P$. pachyrhizi genotypes in the $i$ th pathotype cluster and $N$ is the total number of $P$. pachyrhizi genotypes in the population $A$, which was 52, 35, and 26 in DS, NGS, and SGS zones, respectively. Of the 116 isolates, three were reclassified to the humid forest zone and were not included in this analysis due to the limited sample size. The Shannon index reflects both the number of pathotypes per population and the relative evenness of their frequencies (17).

\section{RESULTS}

Prevalence and severity of soybean rust in agroecological zones. Soybean rust prevalence and severity differed significantly $(P<0.05)$ between agroecological zones during the study period. The disease was most prevalent in the DS zone and disease prevalence was significantly $(P=0.0485)$ higher in this zone than in the SGS zone (Table 1). Disease prevalence in the NGS zone was intermediate but was not significantly different from that in DS or SGS zones. Similarly, soybean rust was most severe in the DS zone but least severe in the NGS zone. Compared to disease prevalence, soybean rust severity was significantly $(P=0.0159)$ higher in the DS zone than in the NGS zone. Disease severity in the SGS zone was intermediate and significantly higher than that in the NGS but was not significantly different from that in the DS zone. Soybean rust severity in the DS zone was approximately three times as higher as that observed in the NGS zone, while rust severity in the SGS zone was about twice as higher as that observed in the NGS zone.

Disease severity exhibited variations within geographical locations across the years of study (Fig. 1). Generally, higher levels of disease severity were observed in soybean fields in DS (Benue and Osun states) and SGS (northern part of Nasarawa state), while low levels of disease severity were observed in NGS. Intermediate levels of disease severity were observed in the DS zone (Ogun and Ondo states). Standardized semivariograms indicated that disease severity levels in different fields were spatially correlated during the study period albeit with variations among years (Fig. 2). In the survey conducted in 2004 when disease level was low (mean severity $25.8 \%$ ), semivariance of disease severity did not increase much as distance increased. However, in 2005 and 2006 surveys when the mean disease severity was 32.5 and $35.5 \%$, respectively, the semivariograms showed the spatial dependence across the range of the surveyed area with values of 75 and $120 \mathrm{~km}$ in respective years. For the interpolated rust severity spatial grids in the top half of the map in Figure 1, the exponential variogram models provided significant fits to empirical variograms in $2005\left(R^{2}=0.52, P<0.0001\right)$ and $2006\left(R^{2}=0.76, P<\right.$ 0.0001 ), but not in 2004 (Fig. 2). For the significant exponential models, the nugget values were 1.1 and 4.5 in 2005 and 2006, respectively. The lower nugget value in 2006 indicates that the degree of spatial correlation was higher during that year than in

TABLE 1. Prevalence and severity of soybean rust based on disease surveys conducted from 2004 to 2006 in three agroecological zones in Nigeria

\begin{tabular}{lcc}
\hline & \multicolumn{2}{c}{ Disease variable } \\
\cline { 2 - 3 } Agroecological zone & Prevalence $(\%)^{\mathrm{a}}$ & Severity $(\%)^{\mathrm{b}}$ \\
\hline Derived savanna & 84.7 & 44.5 \\
Southern Guinea savanna & 56.6 & 33.0 \\
Northern Guinea savanna & 70.8 & 16.3 \\
Least significant difference $(\alpha=0.05)$ & 25.8 & 14.9 \\
\hline
\end{tabular}

${ }^{a}$ Disease prevalence denotes the percentage of fields in which soybean rust symptoms were observed relative to the total number of fields surveyed in each agroecological zone.

${ }^{\mathrm{b}}$ Disease severity refers to leaf area infected (\%) with soybean rust based on a sample of 20 plants in each of the fields.
2005 (Fig. 2). There was no sill for fields within $75 \mathrm{~km}$ in 2006 compared to 2005 where the sill was about 6.3. For the two lower interpolated rust severity grids shown in Figure 1, nugget values and levels of spatial dependence were similar between years to

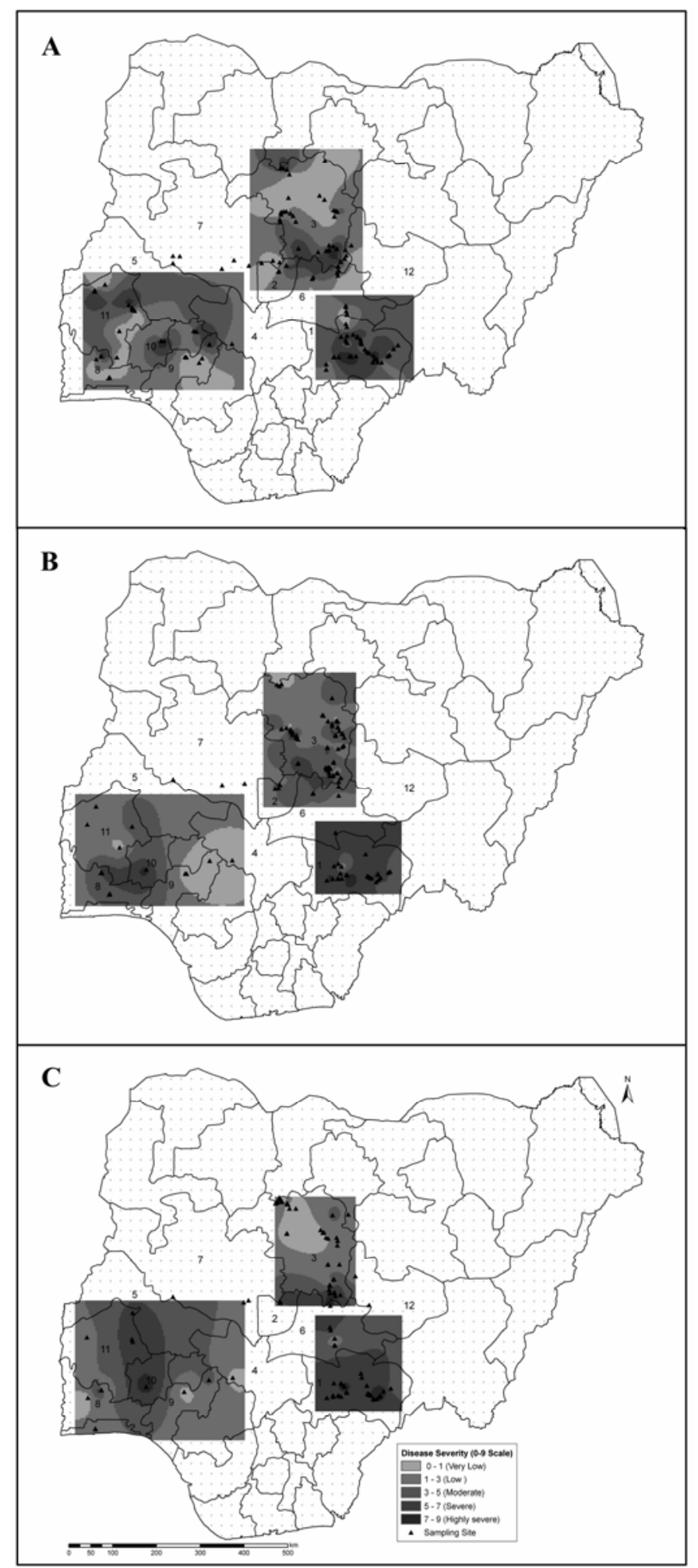

Fig. 1. Spatial grids of interpolated soybean rust (Phakopsora pachyrhizi) severity in three agroecological zones in Nigeria based on field surveys conducted in 2004 (A), 2005 (B), and 2006 (C). Interpolated severity grids were obtained by kriging and are delimited to within a $10-\mathrm{km}$ radius around infected fields observed during the survey. 1 = Benue, $2=$ Federal Capital Territory, $3=$ Kaduna, $4=$ Kogi, $5=$ Kwara, $6=$ Nasarawa, $7=$ Niger, $8=$ Ogun, $9=$ Ondo, $10=$ Osun, $11=$ Oyo, and $12=$ Plateau state. 
those reported for the spatial grids in the top half of the map (data not shown).

Pathogenic variation. Inoculation of the putative differentials with $P$. pachyrhizi isolates resulted in formation of discrete lesions on all the genotypes except for accession PI 594538A on which an HR reaction was observed with no lesion formation. Host genotypes also exhibited TAN, RB, and mixed reactions
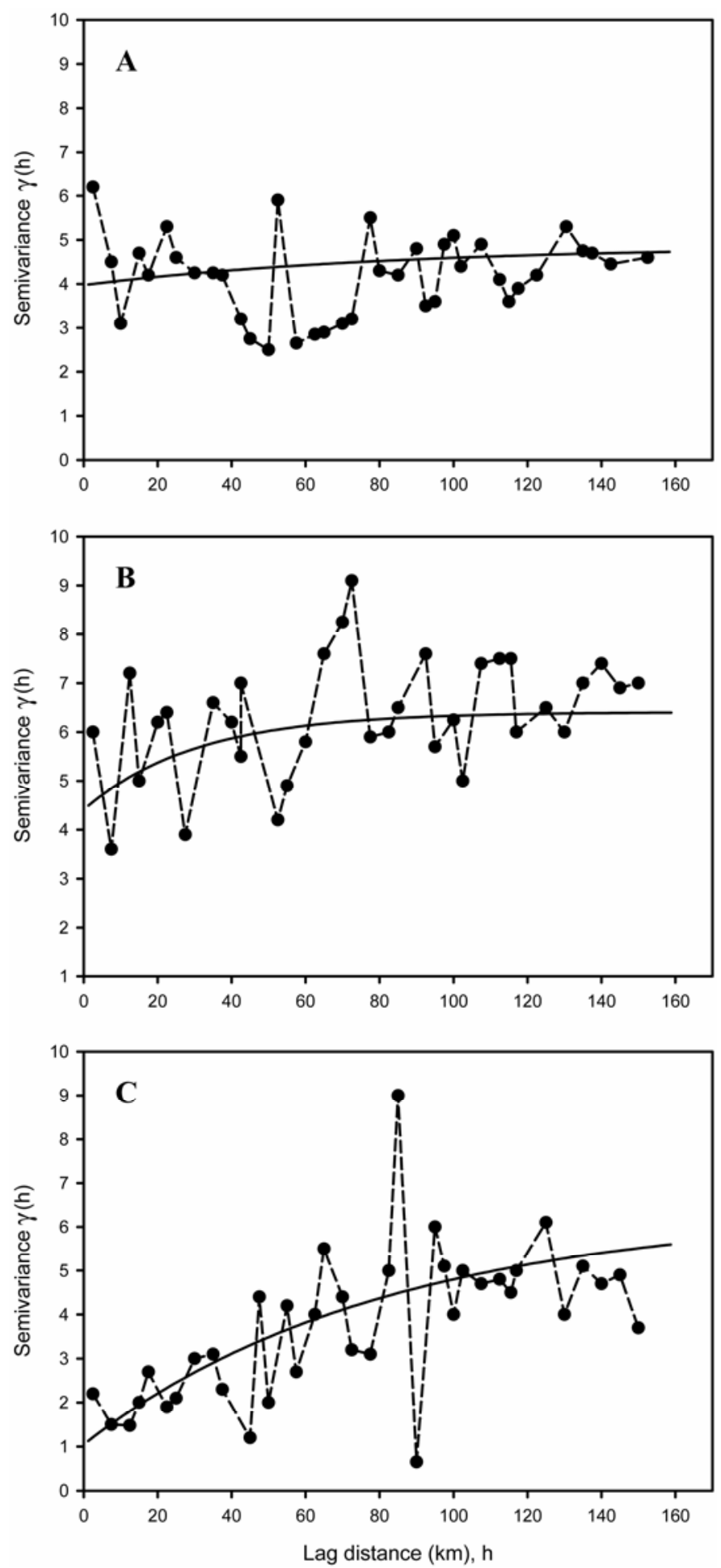

Fig. 2. Semivariograms depicting the spatial dependence among soybean fields infected with soybean rust (Phakopsora pachyrhizi) in Nigeria in 2004 (A), 2005 (B), and 2006 (C). Analysis is based on rust severity (\% leaf area infected) from field surveys in respective years that generated the spatial grid in the top half of each map in Figure 1. Broken lines with closed symbols represent the empirical semivariance values while continuous lines are predicted semivariance values from the fitted exponential variogram model. following inoculation. For example, all isolates resulted in an RB reaction on PI 462312 and most isolates (>92\%) evoked an RB reaction on UG-5, while all isolates resulted in a TAN reaction on TG× 1485-1D (Fig. 3). On infected genotypes, lesions with varying numbers of uredinia per square centimeter were produced on the leaves. On less susceptible genotypes, number of uredinia per unit areas ranged from 1 uredinium to 23 uredinia per $\mathrm{cm}^{2}$. In more susceptible genotypes, the numbers of uredinia were much higher with up to 65 uredinia per $\mathrm{cm}^{2}$.

The eigenvalues for the principal components of the selected isolates showed that the first three components explained $84 \%$ of the total variance (Table 2), indicating that three dimensions were required to explain the variation among the differential lines. The first eigenvector had a positive weight on each component. Variation in this component reflected changes in the overall level of number of uredinia per square centimeter across all differentials, rather than variation in the pattern of number of uredinia per square centimeter over all differentials. The second eigenvector had a large positive coefficient for TG $\times 1844-4 \mathrm{~F}$ and negative coefficient for accession PI 459025B indicating the differential susceptibility of the breeding line and the accession with $R p p_{4}$ resistance gene. Similarly, the third eigenvector can be regarded as contrasting reactions obtained on the highly susceptible breeding line TG× 1485-1D and PI 200492 (with $R p p_{1}$ gene).

Based on the stress statistic, genotypes with minimum stress were eliminated one at a time in a stepwise analysis. Removal of four genotypes did not make any difference in the stress statistic but dropping of the 5th genotype sharply increased the stress statistic. This indicated that four out of the eight genotypes could be discarded. The four genotypes that could be retained were TGX 1485-1D, TG× 1844-4F, PI 459025B, and PI 200492. The contribution of these genotypes towards isolate discrimination was further examined by plotting the selected isolates in space of the first two principal components computed for the eight genotypes after dropping only one, and again after dropping the four genotypes (data not shown). There was a strong agreement between configuration based on eight genotypes and the reduced set of four genotypes, indicating that little information on the first two principal components had been lost by discarding the four genotypes.

From the principal component analysis conducted on number of uredinia per square centimeter matrix of the 116 isolates and eight differentials, the first three and four principal components explained $>80$ and $>90 \%$ of the variations, respectively. Only four

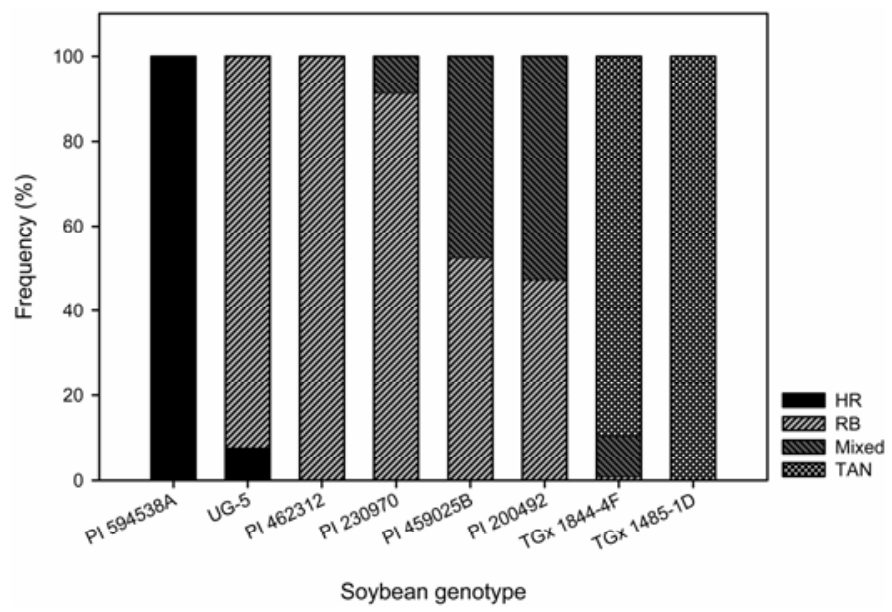

Fig. 3. Frequency of infection types on soybean genotypes inoculated with 116 Phakopsora pachyrhizi isolates collected in Nigeria. HR, RB, and TAN refer to hypersensitive, red-brown and tan lesion reaction type, respectively. Mixed refers to lesions presence of both RB and TAN reaction on the same leaf. The accessions PI 200492, PI 230970, PI 462312, and PI 459025B contain $R p p_{1}, R p p_{2}, R p p_{3}$, and $R p p_{4}$ resistance genes, respectively. 
of the eight putative differentials, TG× 1485-1D, TG× 1844-4F, PI 459025B, and PI 200492, had large positive or negative loadings on the first four principal components. Thus, the variation among the 116 isolates could be adequately explained by these four differentials. Based on cluster analysis on the 116 rust isolates, incremental improvement in $R^{2}$ was more pronounced $(18 \%)$ as the number of clusters increased from two to three (Fig. $4)$. For each additional cluster between three and seven, $R^{2}$ increased by 4.8 and $7 \%$. Above seven clusters, the incremental rise in $R^{2}$ with each additional cluster was less than $4 \%$. Thus, seven clusters were selected using $R^{2}>4.8 \%$ as the threshold to represent the natural groups among the 116 isolates. These seven clusters accounted for $79.9 \%$ of the variation. Similarly, principal component analysis revealed seven pathotype clusters among the 116 isolates (Fig. 5) with the first and second principal components accounting for $67 \%$ of the standardized variance. The characteristics of the isolates in the seven clusters are summarized using mean number of uredinia observed on the eight differentials (Table 3). Isolates in pathotype cluster III were the most virulent across all host differentials. Isolates in clusters V and VI constituted moderately virulent isolates than those in cluster III but were more virulent on PI 200492 than isolates in all other pathotype clusters. Isolates in clusters IV and VII were weakly virulent but cluster VII isolates were more virulent on PI 459025B than cluster IV isolates; both clusters IV and VII isolates did not cause any disease on UG-5. None of the isolates were virulent on PI 594538A.

Geographical distribution of pathotype clusters. Field sites located in the three agroecological zones summarized here represented the source of 113 out 116 P. pachyrhizi examined in this

TABLE 2. Eigenvectors for first three of the eight principal components from an analysis of number of uredinia per square centimeter on eight differential genotypes of soybean inoculated with 116 isolates of Phakopsora pachyrhizi

\begin{tabular}{lccc}
\hline & \multicolumn{3}{c}{ Principal component $^{\mathrm{a}}$} \\
\cline { 2 - 4 } Differential genotype & 1 & 2 & 3 \\
\hline TG× 1485-1D & $\underline{45}$ & $\underline{53}$ & $\frac{-70}{62}$ \\
TG× 1844-4F & $\underline{61}$ & $-\frac{65}{29}$ & 1 \\
PI 459025B & $\underline{62}$ & -30 & 84 \\
PI 200492 & 37 & 67 & 8 \\
Percent cumulative variation explained &
\end{tabular}

a Values have been multiplied by 100 and rounded to the nearest integer, and values greater than the root mean square coefficient are underlined.

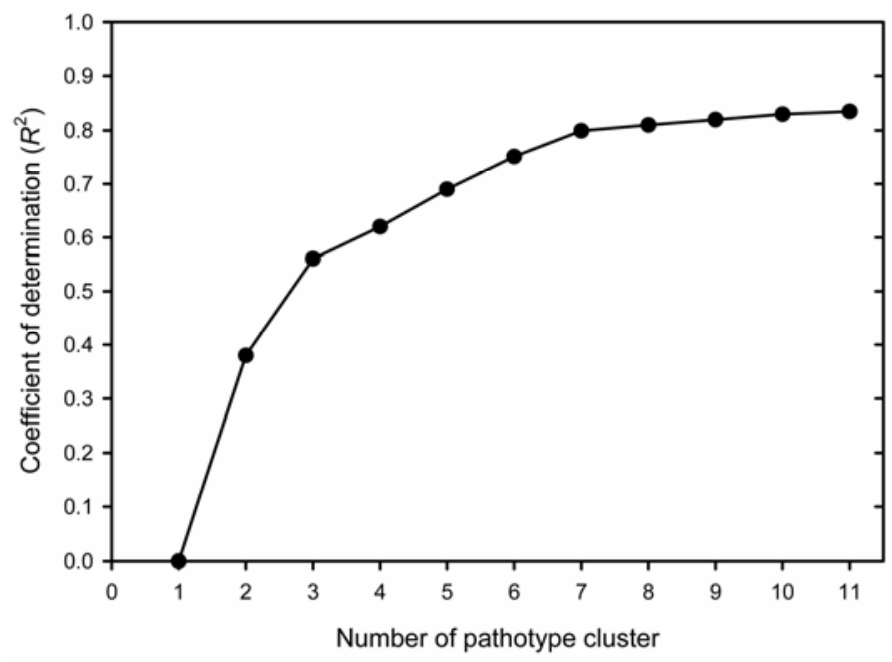

Fig. 4. Cumulative improvement in the coefficient of determination $\left(R^{2}\right)$ when the number of pathotype clusters is increased from 2 to 11 for 116 isolates of Phakopsora pachyrhizi, causing soybean rust on eight differential host genotypes of soybean. study. All the 7 pathotype clusters were found in the DS zone, while pathotype clusters I, II, III, IV, and V were found in the SGS zone (Fig. 6). Pathotype clusters I, II, V, and VI were found in the NGS zone. Shannon's index for diversity revealed a more diverse $P$. pachyrhizi population in the DS and SGS zones, with $H$ values of 1.21 and 1.08 , respectively, compared to the population in NGS zone that had an $H$ value of 0.91 .

\section{DISCUSSION}

This study revealed considerable pathogenic diversity within populations of $P$. pachyrhizi infecting soybean in different geographical areas in Nigeria, and provides the first assessment of pathogen diversity based on a quantitative measure of virulence. Soybean rust was more widespread in the DS agroecological zone with disease incidence being spatially correlated among different surveyed fields. Using multivariate techniques, a differential set consisting of only four genotypes could be useful in classifying $P$. pachyrhizi isolates in Nigeria and seven pathotype clusters were identified to represent the existing range of pathogen virulence.

Both disease prevalence and disease severity within individual fields were higher in the DS zone, while disease severity was the lowest in the NGS agroecological zones. In addition, disease prevalence was also the lowest in the NGS zone during the study period except for data in one year of the survey that resulted in a higher overall prevalence in the NGS zone than in the SGS zone. Further, disease severity in the DS zone was three times higher as that observed in the NGS zone. Two reasons can explain this difference in disease intensity. First, the DS zone has a bimodal rainfall regime (mean $1,500 \mathrm{~mm}$ ) with maximum temperatures ranging between 25 to $30^{\circ} \mathrm{C}$, while the NGS zone is characterized by unimodal distribution (mean $900 \mathrm{~mm}$ ) and temperatures ranging from 30 to $40^{\circ} \mathrm{C}(22)$. Rust epidemics occur when prolonged conditions of leaf wetness and moderate temperatures prevail and

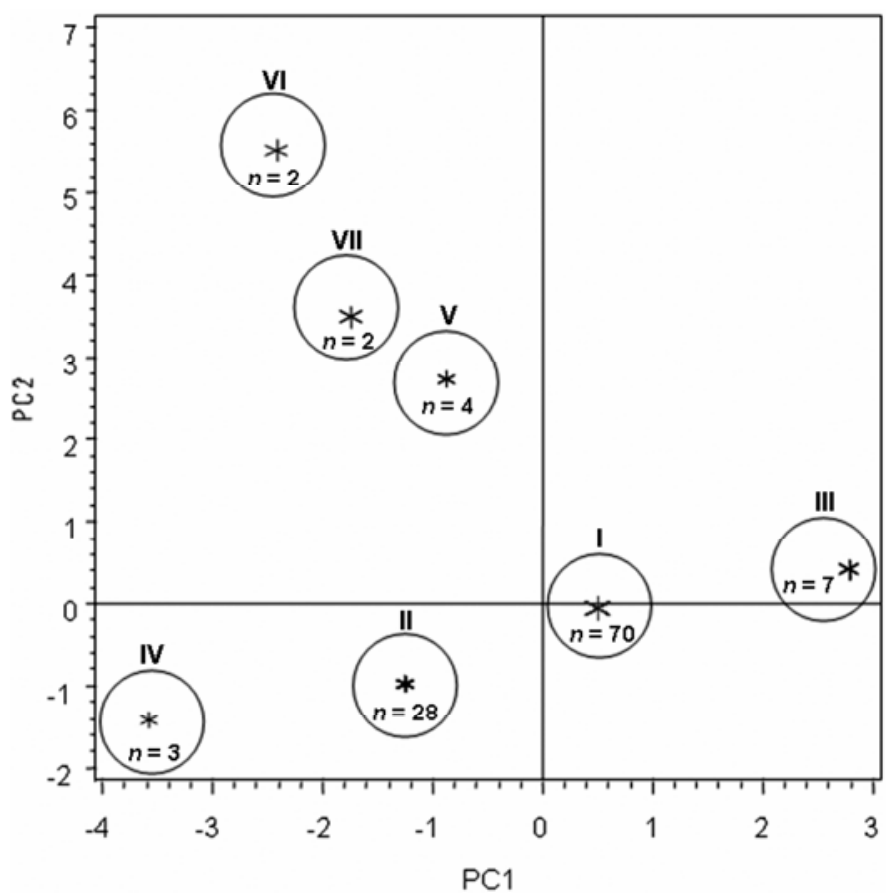

Fig. 5. Results of the principal component and cluster analysis on number of uredinia per square centimeter following inoculation of the soybean differential set with 116 isolates of Phakopsora pachyrhizi. Sample size (n) shown within clusters (circles) represent the number of isolates, plotted in a plane defined by the first two principal components $(\mathrm{PC} 1 \times \mathrm{PC} 2)$. Roman numerals adjacent to each cluster are the pathotype cluster groupings described in the main text. 
epidemics are retarded under dry conditions and when temperatures are $>30^{\circ} \mathrm{C}$. Thus, the moderate temperatures and high rainfall that usually prevail in the DS zone favor development of rust epidemics. Secondly, most of the soybean production in Nigeria is concentrated in the DS zone where several edible and wild legumes such as kudzu (Pueraria lobata), which are potential alternative hosts for $P$. pachyrhizi, thrive even in the dry season (32). Thus, although the sources of inoculum have not been documented in West Africa, it is conceivable that these wild and edible legumes may serve as bridging hosts for inoculum increase and spread of soybean rust in the DS zone.

Different semivariograms were observed in the 3 years of study, and in survey years when rust severity was high, with semivariances increasing with distance between sampled fields. The range of spatial influence in the years with high disease severity was approximately 75 to $120 \mathrm{~km}$. This range of influence is similar to that reported for the spread of soybean rust in the southeastern United States (12). The long range of spatial influence is in line with the relatively high critical doses of solar radiation for $P$. pachyrhizi spores as compared to fungal spores that have a much short range such those of Bremia lactucae (36). Inoculum availability, host age, and environmental factors such as wind, moisture, and solar radiation influence the spread of soybean rust. Thus, a possible explanation for the different semivariograms in the different years may be related to inoculum availability and environmental factors. Since fields were sampled when plants were at R6 and the same fields were not sampled during the survey period, differences in soybean rust within a field could be mainly due to inoculum availability. However, the differences between surveys from one year to the next may be due to environmental factors. Thus, the exact range of spatial influence of soybean rust cannot be determined since the semivariograms had very different sill levels. Nonetheless, the interpolated disease maps illustrate the spatial spread of soybean rust in Nigeria. Using this GIS analysis, we showed that specific areas in DS (mainly in Benue and Ogun states) and SGS (in Nasarawa state) are conducive to the spatial spread of soybean rust epidemics while most areas located in NGS zones are less favorable.

Diversity in $P$. pachyrhizi has primarily been based on qualitative assessment of virulence using RB/TAN reactions types. A major disadvantage of this approach is that virulence is clearly not a qualitative phenomenon (28) as sufficiently large numbers of pathogen isolates can reasonably be expected to show a continuous range of capacity to damage the host. In a recent study, Bonde et al. (4) observed for example that the RB reaction may be a spectrum of reaction types. Further, they also observed that, whereas the reaction phenotypes on the original sources were easy to score, it was very difficult to ascertain whether some lines derived from them had RB or TAN lesions and concluded that reactions need to be measured quantitatively. In this study, we examined diversity based on a quantitative assessment of virulence using the number of uredinia per unit leaf tissue. This quantitative assessment enabled us to delineate differences between rust isolates using the host differential set than would have been possible otherwise.
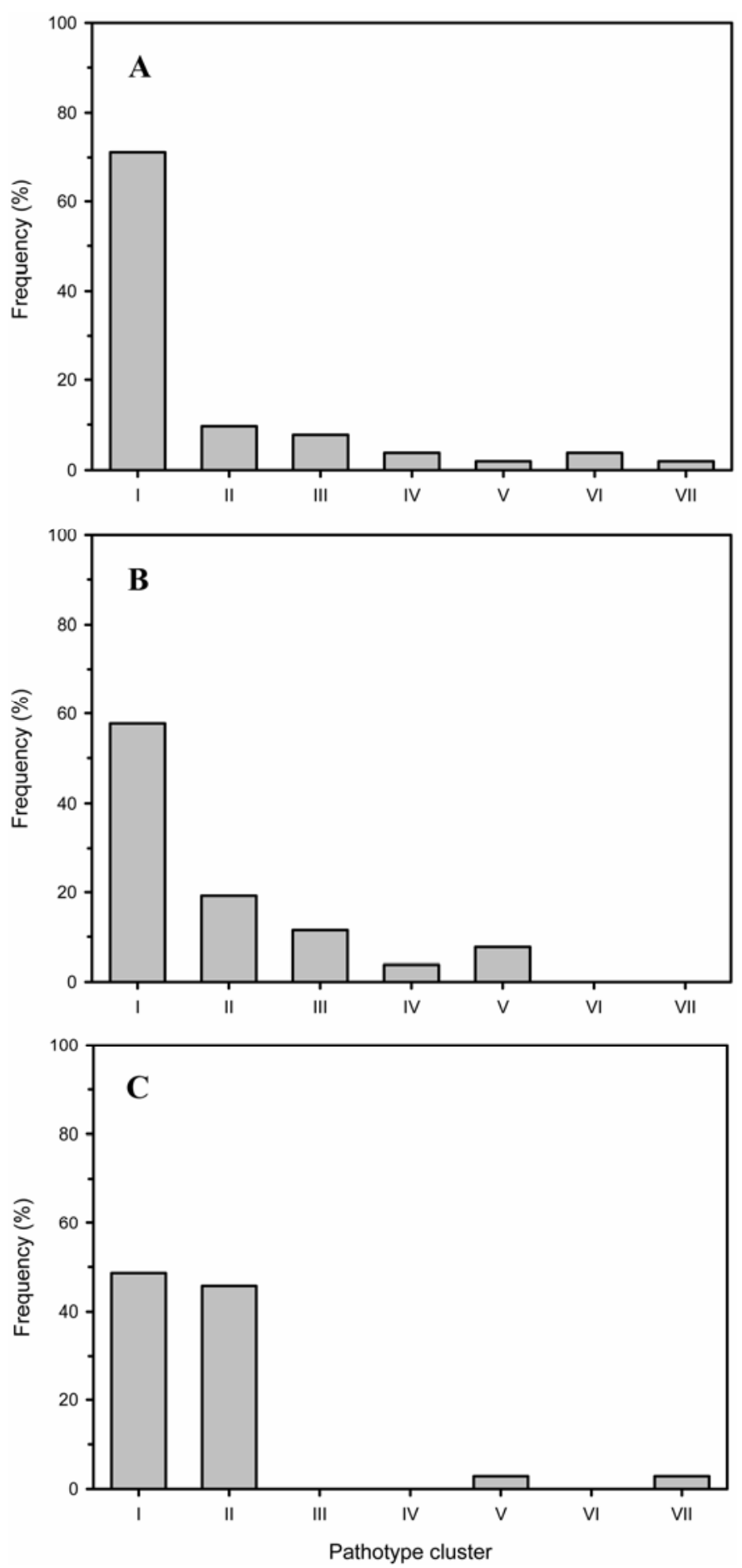

Fig. 6. Frequency of Phakopsora pachyrhizi isolates in seven pathotype clusters obtained from soybean in three agroecological zones in Nigeria: A, Derived savanna, B, Southern Guinea savanna, and C, Northern Guinea savanna zones.

TABLE 3. Mean and standard deviation (in parenthesis) of number of uredinia on a set of eight differential genotypes of soybean for 116 Nigerian field isolates of Phakopsora pachyrhizi in seven groupings derived by cluster analysis

\begin{tabular}{|c|c|c|c|c|c|c|c|c|c|}
\hline \multirow[b]{2}{*}{ Pathotype cluster } & \multirow[b]{2}{*}{ Number of isolates } & \multicolumn{8}{|c|}{ Uredinia per $\mathrm{cm}^{2}$} \\
\hline & & $\mathrm{TG} \times 1485-1 \mathrm{D}$ & $\mathrm{TG} \times 1844-4 \mathrm{~F}$ & PI 459025B & PI 200492 & PI 230970 & PI 462312 & UG-5 & PI 594538A \\
\hline I & 70 & $58.7(6.3)$ & $38.2(5.3)$ & $13.1(2.2)$ & $13.0(3.2)$ & $9.2(2.1)$ & $7.2(3.2)$ & $3.2(2.4)$ & 0.0 \\
\hline IV & 3 & $32.7(8.8)$ & $16.8(0.3)$ & $8.1(5.4)$ & $11.2(1.6)$ & $5.8(5.1)$ & $4.4(3.8)$ & $0.0(0.0)$ & 0.0 \\
\hline $\mathrm{V}$ & 4 & $56.1(6.2)$ & $40.3(4.0)$ & $13.1(2.8)$ & $21.4(4.8)$ & $11.1(3.7)$ & $7.7(2.1)$ & $4.6(2.0)$ & 0.0 \\
\hline VI & 2 & $59.0(1.9)$ & $31.8(7.4)$ & $19.9(0.2)$ & $22.1(2.3)$ & $13.1(1.9)$ & $7.7(0.0)$ & $4.5(0.9)$ & 0.0 \\
\hline
\end{tabular}


From an initial set of eight putative differentials, a reduced set comprising four genotypes could adequately explain the pathogenic variation among $P$. pachyrhizi in Nigeria. Within this reduced set of host differentials, TG× 1485-1D and TG× 1844-4F are breeding lines with high and moderate susceptibility to rust, respectively, while the accessions PI 200492 and 459025B possess the $R p p_{1}$ and $R p p_{4}$ resistance genes, respectively. Both PI 200492, and 459025B were part of the differential hosts recommended by AVRDC (33) and a differential set proposed for identification of rust races in Japan (37). In the latter studies, both PI 200492 and 459025B were susceptible to most of the rust isolates in Japan (only 4 out of 22 isolates resulted in a resistant reaction). Both accessions were moderately resistant to $P$. pachyrhizi isolates in the present study. Differences in the reaction of PI 200492 and $459025 \mathrm{~B}$ in these studies are primarily due to the variability of the fungus within respective countries. Variation in virulence among isolates from the same field and those collected from different geographical areas has been reported (30). Using isolates from South Africa, the AVRDC recommended differential lines were all found to be susceptible to $P$. pachyrhizi (8). Thus, without a useful host differential set that uses key informative genotypes, it may not be possible to document information on the structure and dynamics of pathogen races following the release of new cultivars. The differential set described in this study is a valuable tool for additional work on pathogen population and dynamics not only in Nigeria, but in other locations in West Africa where rust occurs.

In this study, we identified seven pathotype clusters of $P$. pachyrhizi infecting soybean in Nigeria. Most isolates belonged to clusters I and II, with isolates in cluster I being more virulent than those in cluster II on all the host differentials. In addition, pathotype clusters I and II were the most frequent in all geographical zones. However, the most virulent and least virulent pathotypes were present only in the DS and SGS zones and the greatest diversity in virulence phenotypes was also observed in these two zones. These differences in diversity of $P$. pachyrhizi observed in this study could be explained by the host-pathogen co-evolution within the respective agroecological zones. Most of the soybean production is concentrated in the DS and SGS zones where up to two crops are produced in a year and where growers cultivate a wide range of soybean cultivars (27) without adequate rust resistance. It has been suggested that in the absence of racespecific resistance, host-pathogen co-evolution may favor simple races due to fitness costs associated with virulence (7). Life history traits of $P$. pachyrhizi, such as wide host range and spore transport to long distances, favor rapid distribution of isolates across large geographical areas. Thus, our results point to a diversification in the pathogen population in response to the diversity in host population both soybean and nonsoybean systems in the DS and SGS zones. The limited diversity in NGS is likely to change as soybean production is pushed towards more marginal areas as agronomically adapted cultivars become available for production in the zone (3). Certainly, the number of isolates examined in the present study is relatively small and more extensive sampling over several seasons is necessary for a more realistic examination of co-evolution.

Of the eight putative differentials used in this study, a reduced set consisting of TG× 1485-1D, TG× 1844-4F, PI 459025B, and PI 200492 was determined to be useful in differentiating pathogenic variation among our isolates. Nonetheless, the range of pathogenic variation that was captured by the differential hosts was still limited. Theoretically, if each of the eight putative differentials carried a unique resistance factor against each of the pathogen isolates tested, up to $2^{8}$ pathotypes clusters could be differentiated (11). This difference between observed and hypothesized pathogenic variability can be explained partly by the choice of host differentials. Differentials other than TG× 14851D, TG× 1844-4F, PI 459025B, and PI 200492 may not have been useful in differentiating the isolates as supported by our data analysis. Similar differences between observed and hypothesized pathogenic variability have previously been reported in other host-pathogen systems (10). Ideally, a more universal host differential set should consist of isolines of characterized genes for resistance to avoid characterizing unnecessary virulence carried by the pathogen that is not needed to facilitate pathogenicity in the cultivars of interest. The reduced set of differential hosts reported in this study represents some of the resistances in use in Nigeria although a clear understanding of the genetics of resistance is lacking for the released cultivars, TG× 1485-1D and TG× 1844-4F, and resistance donors, PI 594538A and UG-5, currently being used in the IITA soybean breeding program (1). The latter two accessions are good sources of resistance due to their resistance to all $P$. pachyrhizi isolates from Nigeria.

Future research efforts should focus in identifying pathogenic clusters in different regions in major soybean areas in West Africa using the host differential identified in this work or using a more expanded differential set. This information can then be used to target cultivars with specific resistance to match prevailing pathotype groups in respective regions. With increased wide spread production of locally adapted cultivars, pathogenic diversity is expected to intensify. Four of the eight putative differentials are being used to generate genotypes for possible cultivar development. Although the reduced set of four differentials adequately described the pathogenic variation in the isolate collection in this study, we recommend the use of all eight putative differentials to facilitate comparison between existing and new pathotypes that may evolve in the future. As pathogenic data on additional isolates becomes available, the number and structure of the pathotype clusters will become clearer.

\section{ACKNOWLEDGMENTS}

This research was funded by the United States Agency for International Development as a linkage grant to IITA and USDA-ARS.

\section{LITERATURE CITED}

1. Adeleke, R. A., Asafo-Adjei, B., Twizeyimana, M., Ojiambo, P. S., Paul, C., Hartman, G. L., Dashiell, K., and Bandyopadhyay, R. 2006. Breeding for rust resistance in soybean at the International Institute of Tropical Agriculture, Nigeria. In: National Soybean Rust Symposium, St. Louis, MO.

2. Akinsanmi, O. A., Ladipo, J. L., and Oyekan, P. O. 2001. First report of soybean rust (Phakopsora pachyrhizi) in Nigeria. Plant Dis. 85:97.

3. Amaza, P. S., and Ogundari, K. 2008. An investigation of factors that influence the technical efficiency of soybean production in the Guinea savannas of Nigeria. J. Food Agric. Env. 6:132-136.

4. Bonde, M. R., Nester, S. E., Austin, C. N., Stone, C. L., Frederick, R. D., Hartman, G. L., and Miles, M. R. 2006. Evaluation of virulence of Phakopsora pachyrhizi and P. meibomiae isolates. Plant Dis. 90:708-716.

5. Bromfield, K. R. 1984. Soybean Rust. Monogr. No. 11. American Phytopathological Society, St. Paul, MN.

6. Bromfield, K. R., Melching, J. S., and Kingsolver, C. H. 1980. Virulence and aggressiveness of Phakopsora pachyrhizi isolates causing soybean rust. Phytopathology 70:17-21.

7. Burdon, J. J. 1993. The structure of pathogen populations in natural plant communities. Annu. Rev. Phytopathol. 31:305-323.

8. Caldwell, P. M., Govender, P., and Laing, M. D. 2003. Epidemiology, electron microscopy and other initial investigations on soybean rust (Phakopsora pachyrhizi). Page 42 in: Proceedings of the 41st Annual Congress of the Southern African Society for Plant Pathology, Bain's Game Lodge, Bloemfontein.

9. Campbell, C. L., and Madden, L. V. 1990. Introduction to Plant Disease Epidemiology. John Wiley and Sons, New York.

10. Chakraborty, S., Fernandes, C. D., Charchar, M. J. d'A., and Thomas, M. R. 2002. Pathogenic variation in Colletotrichum gloeosporioides infecting Stylosanthes spp. in a center of diversity in Brazil. Phytopathology 92:553-562.

11. Chakraborty, S., Thomas, M. R., and Ellis, N. 1996. A multivariate analysis of pathogenic variation in Colletotrichum gloeosporioides infecting the tropical pasture legume, Stylosanthes scabra. Phytopathology 86:283-289. 
12. Christiano, R. C. S., and Scherm, H. 2007. Quantitative aspects of the spread of Asian soybean rust in the southeastern United States, 2005 to 2006. Phytopathology 97:1428-1433.

13. ESRI, 2003. ArcGIS 9: Using ArcGIS Geostatistical Analyst. ESRI Press, Redlands, CA.

14. FAO. 2006. Food and Agricultural Organization. http://faostat.fao.org.

15. Frederick, R. D., Snyder, C. L., Peterson, G. L., and Bonde, M. R. 2002. Polymerase chain reaction assays for the detection and discrimination of the soybean rust pathogens Phakopsora pachyrhizi and P. meibomiae. Phytopathology 92:217-227.

16. Gomez, K. A., and Gomez, A. A. 1984. Statistical Procedures for Agricultural Research, 2nd ed. John Wiley and Sons, New York.

17. Grünwald, N. J., Goodwin, S. B., Milgroom, M. G., and Fry, W. E. 2003. Analysis of genotypic diversity data for populations of microorganisms. Phytopathology 93:738-746.

18. Hartman, G. L., Miles, M. R., and Frederick, R. D. 2005. Breeding for resistance to soybean rust. Plant Dis. 89:664-666.

19. Hymowitz, T., and Shurtleff, W. R. 2005. Debunking soybean myths and legends in the historical and popular literature. Crop Sci. 45:473-476.

20. Kawuki, E., Adipala, E., and Tukamuhabwa, P. 2003. Yield loss associated with soybean rust (Phakopsora pachyrhizi Syd.) in Uganda. J. Phytopathol. 151:7-12.

21. Kochman, J. K. 1977. Soybean rust in Australia. Pages 44-48 in: Rust of soybean - The Problem and Research Needs. INTSOY Series No. 12. R. E. Ford and J. B. Sinclair, eds. University of Illinois, Urbana.

22. Kowal, J. M., and Kassam, A. H., 1978. Agricultural Ecology of Savanna. A Study of West Africa. Clarendon Press, Oxford, UK.

23. Miles, M. R., Frederick, R. D., and Hartman, G. L. 2006. Evaluation of soybean germplasm for resistance to Phakopsora pachyrhizi. Online. Plant Health Progress doi:10.1094/PHP-2006-0104-01-RS.

24. Mueller, T. A., Miles, M. R., Hartman, G. L., and Morel, W. 2007. Evaluations of fungicides and fungicides timing for the control of soybean rust at Pirapo, Paraguay, 2005-2006. Plant Dis. Manag. Rep. (online) Report 1:FC063. DOI:10.1094/PDMR01. American Phytopathological Society Press, St. Paul, MN.

25. Parlevliet, J. E. 1979. Components of resistance that reduce the rate of epidemic development. Annu. Rev. Phytopathol. 17:203-222.

26. Ramstedt, M., Hurtado, S., and Åström, B. 2002. Pathotypes of Melampsora rust on Salix in short-rotation forestry plantations. Plant Pathol. 51:185-190.

27. Sanginga, N., Dashiell, K. E., Diels, J., Vanlauwe, B., Lyasse, O., Carsky,
R. J., Tarawali, S., Asafo-Adjei, B., Menkir, A., Schulz, S., Singh, B. B., Chikoye, D., Keatinge, D., and Ortiz, R. 2003. Sustainable resource management coupled to resilient germplasm to provide new intensive cereal-grain-legume-livestock systems in the dry savanna. Agr. Ecosyst. Environ. 100:305-314

28. Shaner, G., Stromberg, E. L., Lacy, G. H., Barker, K. R., and Pirone, T. P. 1992. Nomenclature and concepts of pathogenicity and virulence. Annu. Rev. Phytopathol. 30:47-66.

29. Shannon, C. E., and Weaver, W. 1949. The Mathematical Theory of Communication. University of Illinois Press, Urbana, IL.

30. Shin, D. C., and Tschanz, A. T. 1986. Studies on physiological reactions of soybean cultivars tolerant and susceptible to rust (Phakopsora pachyrhizi Syd.). Korean J. Crop Sci. 31:440-446.

31. Sinclair, J. B., and Hartman, G. L. 1999. Soybean rust. Pages 25-26 in: Compendium of Soybean Diseases. G. L. Hartman, J. B. Sinclair, and J. C. Rupe, eds. American Phytopathological Society, St. Paul, MN.

32. Tian, G., Kolawole, G. O., Ishida, F., and Carsky, R. J. 2000. On-farm study of Pueraria cover-crop fallow system in the derived savanna of West Africa. Pages 185-193 in: Cover Crops for Natural Resource Management in West Africa. R. J. Carsky, A. C. Etèka, J. D. H. Keatinge, and V. M. Manyong, eds. International Institute of Tropical Agriculture, Ibadan, Nigeria.

33. Tschanz, A. T., and Shanmugasundaram, S. 1985. Soybean rust. Pages 562-567 in: Proceedings of the 3rd World Soybean Research Conference. R. Shibles, ed. Ames, IA.

34. Twizeyimana, M., Ojiambo, P. S., Ikotun, T., Ladipo, J. L., Hartman, G. L., and Bandyopadhyay, R. 2008. Evaluation of soybean germplasm for resistance to soybean rust (Phakopsora pachyrhizi) in Nigeria. Plant Dis. 92:947-952.

35. Twizeyimana, M., Ojiambo, P. S., Ikotun, T., Paul, C., Hartman, G. L., and Bandyopadhyay, R. 2007. Comparison of field, greenhouse, and detached-leaf evaluations of soybean germplasm for resistance to Phakopsora pachyrhizi. Plant Dis. 91:1161-1169.

36. Wu, B. M., van Bruggen, A. H. C., Subbarao, K. V., and Pennings, G. G. H. 2001. Spatial analysis of lettuce downy mildew using geostatistics and geographic information systems. Phytopathology 91:134-142.

37. Yamaoka, Y., Fujiwara, Y., Kakishima, M., Katsuya, K., Yamada, K., and Hagiwara, H. 2002. Pathogenic races of Phakopsora pachyrhizi on soybean and wild host plants collected in Japan. J. Gen. Plant Pathol. 68:52-56.

38. Yeh, C. C. 1983. Physiological races of Phakopsora pachyrhizi in Taiwan. J. Agric. Res. China 32:69-74. 